\title{
On Throughput Efficiency of Geographic Opportunistic Routing in Multihop Wireless Networks.
}

\author{
Kai Zeng Wenjing Lou Jie Yang D. Richard Brown III \\ Department of ECE \\ Worcester Polytechnic Institute \\ Worcester, MA 01609 \\ \{kzeng, wjlou, abbyyang, drb\}@wpi.edu
}

\begin{abstract}
Geographic opportunistic routing (GOR) is a new routing concept in multihop wireless networks. In stead of picking one node to forward a packet to, GOR forwards a packet to a set of candidate nodes and one node is selected dynamically as the actual forwarder based on the instantaneous wireless channel condition and node position and availability at the time of transmission. GOR takes advantages of the spatial diversity and broadcast nature of wireless communications and is an efficient mechanism to combat the unreliable links. The existing GOR schemes typically involve as many as available next-hop neighbors into the local opportunistic forwarding, and give the nodes closer to the destination higher relay priorities. In this paper, we focus on realizing GOR's potential in maximizing throughput. We start with an insightful analysis of various factors and their impact on the throughput of GOR, and propose a local metric named expected one-hop throughput (EOT) to balance the tradeoff between the benefit (i.e., packet advancement and transmission reliability) and the cost (i.e., medium time delay). We identify an upper bound of EOT and proof its concavity. Based on the EOT, we also propose a local candidate selection and prioritization algorithm. Simulation results validate our analysis and show that the metric EOT leads to both higher one-hop and path throughput than the corresponding pure GOR and geographic routing.
\end{abstract}

\section{Categories and Subject Descriptors}

C.2.2 [Computer-Communication Networks]: Network Protocols-Routing Protocols; C.2.1 [Computer-Communication Networks]: Network Architecture and Design Wireless Communication

*This work was supported in part by the US National Science Foundation under grants CNS-0626601 and CCF0447743 .

Permission to make digital or hard copies of all or part of this work for personal or classroom use is granted without fee provided that copies are not made or distributed for profit or commercial advantage and that copies bear this notice and the full citation on the first page. To copy otherwise, to republish, to post on servers or to redistribute to lists, requires prior specific permission and/or a fee.

Qshine'07 August 14-17, 2007, Vancouver, Canada

Copyright 2007 ACM 978-1-59593-756-8 ...\$5.00.

\section{General Terms}

Algorithms, Performance

\section{Keywords}

wireless, geographic, performance

\section{INTRODUCTION}

Routing in multihop wireless networks is a challenging issue. The main difficulty lies in that wireless links can be very unstable and unreliable $[4,15]$. Traditional routing protocols for wireless networks have followed the routing concept in wired networks by abstracting wireless links as wired links, and focused on finding a fixed shortest path for forwarding packets between a pair of nodes. However, it is not an ideal approach for wireless networks with broadcast links of time varying qualities. Recently, a new routing paradigm, known as opportunistic routing $[18,11,3]$ (or contention-based forwarding [6]), was proposed to cope with the unreliability of link quality.

The basic idea behind opportunistic routing is to integrate the network and MAC layers such that at the network layer a set of forwarding candidates are selected and at the MAC layer one node is chosen as the actual relay. Owing to the broadcast nature and spatial diversity of the wireless medium, the probability of at least one forwarding candidate correctly receiving the packet will increase when multiple candidates are involved, thus improve the packet delivery efficiency such as throughput $[3,6]$ or energy efficiency [11, $18,13]$.

Two important issues of opportunistic routing are forwarding candidates selection and relay priority assignment. Several variants of opportunistic routing $[18,11,6]$ leverage the location information of nodes to select forwarding candidates and prioritize them. For example, in [18], all the available next-hop neighbors that are nearer than the sender to the destination are selected as the candidates, and the nodes closer to the destination are given higher relay priorities. In this paper, we mainly focus on this kind of geographic opportunistic routing (GOR).

Intuitively, giving nodes closer to the destination higher relay priorities will maximize the expected packet advancement [13]. However it is not always the case to maximize the throughput, especially when the packet reception ratios from the sender to the neighbors that make large advancements are low. Since before relaying the packet, lower-priority candidates always need to wait for a certain period of time to confirm that higher-priority candidates have not relayed the 
packet, it will introduce larger latency when higher-priority candidates are very unlikely to receive the packet correctly. On the other hand, it is also not a good strategy to involve as many as possible next-hop nodes as candidates. Although involving more forwarding candidates tends to increase the packet advancement and delivery reliability, the medium time needed for ensuring only one actual forwarder to relay the packet is also expected to increase when more forwarding candidates are involved. So there exists a tradeoff between the medium time [2], which is directly relative to the throughput, and other performance goals, such as packet advancement and delivery reliability. This trade-off is not well studied in the existing works $[18,11,3]$.

In this paper, we endeavor to study the impact of candidate selection, prioritization and coordination on the distance-reliability-time trade-off in GOR. We introduce a local metric, expected one-hop throughput (EOT), to balance these factors. We also derive an upper bound of the EOT, and unveil its concavity, which indicates that the gained throughput becomes marginal when the number of forwarding candidates keeps increasing. Based on EOT, we further propose a heuristic algorithm to select the forwarding candidates and assign relay priority to them. The simulation results validate our analysis and show that the metric EOT leads to both higher one-hop and path throughput than the corresponding pure GOR and geographic routing (GR).

\section{PROBLEM FORMULATION}

\subsection{System Model}

Fig. 1 shows an example of GOR. Assume node $S$, i.e., the sender, is forwarding a packet to a destination $D$, and $s_{i}$ is one of $S$ 's neighbors which are closer to $D$ than $S$. Let $\mathcal{C}$ be the set of $s_{i}$ which is the available next-hop node set of $S$, and let $N=|\mathcal{C}|$, which is the number of nodes in $\mathcal{C}$. Like geographic routing $[10,17,9,14]$, we assume $S$ is aware of the location information of itself, $s_{i}$ 's and $D$. Define $a_{i}$ in Eq. (1) as the packet advancement toward the destination when a packet sent by $S$ is relayed by $s_{i}$.

$$
a_{i}=d(S, D)-d\left(s_{i}, D\right)
$$

where $d(S, D)$ and $d\left(s_{i}, D\right)$ are the Euclidian distances between $S$ and $D$ and between $s_{i}$ and $D$, respectively.

Without loss of generality, we assume all the nodes in $\mathcal{C}$ are indexed from $s_{1}$ to $s_{N}$ in descending order according to the advancement $a_{i}$, i.e., $a_{m} \geq a_{n}, \forall s_{m}, s_{n}$ where $m<n$. Each link from $S$ to $s_{i}$ is associated to a pair, $\left(a_{i}, p_{i}\right)$, where $p_{i}$ is the data packet reception ratio (PRR) from node $S$ to $s_{i}$. A node is a neighbor of $S$ when the PRR from $S$ to it is larger than some non-negligible probability ${ }^{1}$. The PRR information on each link can be obtained by using probe messages $[4,8]$ and is assumed to be independent. Let $\mathcal{F}$ denote the forwarding candidate set of node $S$, which includes all the nodes selected to get involved in the local collaborative forwarding, and $r=|\mathcal{F}|$. Here $\mathcal{F}$ is a subset of $\mathcal{C}$, while in the existing pure opportunistic routing protocols $[18,3], \mathcal{F}=\mathcal{C}$.

The GOR procedure is as follows: node $S$ selects $\mathcal{F}$ based on its knowledge of $\mathcal{C}\left(a_{i}\right.$ 's and $p_{i}$ 's); then broadcasts the data packet to the forwarding candidates in $\mathcal{F}$ after detecting the channel is idle. Candidates in $\mathcal{F}$ follow a specific

\footnotetext{
${ }^{1}$ In this paper, we set the threshold as 0.1 .
}

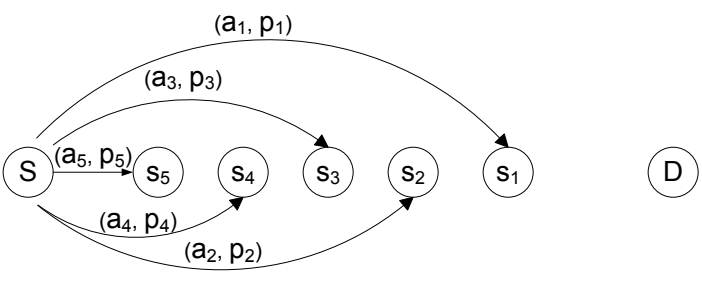

Figure 1: Node $S$ is forwarding a packet to a remote destination $D$.

priority to relay the packet, that is, a forwarding candidate will only relay the packet if it received the packet correctly and all the nodes with higher priorities failed to do so. The actual forwarder will become a new sender and suppress all the other potential forwarders in $\mathcal{F}$. When no forwarding candidate has successfully received the packet, the sender will retransmit the packet if retransmission is enabled. The sender will drop the packet when the retransmissions reach the limit. This procedure reiterates until the packet arrives at the destination.

\subsection{Impact of Candidate Selection, Prioritiza- tion and Coordination on Throughput}

To ensure the relay priority among the forwarding candidates, a MAC protocol similar to those proposed in $[18,6]$ is necessary. For example, a feasible MAC protocol could proceed as following: when the sender decides the $\mathcal{F}$ and detects the channel is idle for a while, it broadcasts the data packet, in which the intended MAC address of the forwarding candidates and their relay priorities are included. To ensure the candidates to follow the priorities to relay the packet, the candidate with $i^{t h}$ priority will wait $(i-1) T_{A C K}\left(T_{A C K}\right.$ is time needed for transmitting an ACK packet) time before it sends out the ACK when it received the packet correctly or keep silent otherwise. Here the ACK message plays two roles, one is for acknowledgement to the sender, the other is for suppressing lower-priority candidates. That is, whenever a lower-priority candidate hears an ACK sent from a higherpriority candidate, it will suppress itself from relaying the packet. In our analysis, we assume the relay priority can be strictly enforced, i.e., for this feasible MAC protocol, the ACK can be correctly received by the sender and the candidates with probability 1 . This assumption is reasonable because typically the ACK packet is small and broadcast at the basic rate, it is unlikely to be lost and can be transmitted correctly for a longer range than the data packet.

We define the one-hop medium time consumed by the $i^{\text {th }}$ candidate as the time slot from the time when the sender is going to broadcast the packet to the time when the $i^{\text {th }}$ candidate claims it receives the packet. Although the medium time for locally forwarding a packet varies for different MAC protocols, for any protocol, it can be divided into two parts. One part is the sender delay and the other part is candidate coordination delay, which are defined as follows:

- $T_{s}$ : the sender delay defined in Eq. (2) which can be further divided into three parts: channel acquisition time $\left(T_{c}\right)$, data transmission time $\left(T_{d}\right)$ and propagation delay $\left(T_{\text {prop }}\right)$.

$$
T_{s}=T_{c}+T_{d}+T_{\text {prop }}
$$




\begin{tabular}{|c|c|}
\hline Parameter & Value \\
\hline Basic Bit Rate (BBR) & $1 \mathrm{Mbps}$ \\
\hline Bit Rate (BR) & $11 \mathrm{Mbps}$ \\
\hline PHY Header Size (PHS) & $192 \mathrm{bits}$ \\
\hline MAC Header Size (MHS) & $272 \mathrm{bits}$ \\
\hline$T_{h}$ & $\mathrm{PHS} / \mathrm{BBR}+\mathrm{MHS} / \mathrm{BR}$ \\
\hline$T_{A C K}$ & $112 / \mathrm{BR}+\mathrm{PHS} / \mathrm{BBR}$ \\
\hline$T_{S I F S}$ & $10 \mu \mathrm{s}$ \\
\hline$T_{D I F S}$ & $50 \mu s$ \\
\hline
\end{tabular}

Table 1: IEEE 802.11 DSSS PHY Parameter Set

For a contention-based MAC protocol (like 802.11), $T_{c}$ is time needed for the sender to acquire the channel before it transmits the data packet, which may include the back-off time, Distributed Interframe Space (DIFS) and time for transmitting Ready-To-Send (RTS) packet. $T_{d}$ is equal to protocol heads transmission time plus data payload transmission time, which is

$$
T_{d}=T_{h}+T_{p l}
$$

$T_{\text {prop }}$ is the time for the signal propagating from the sender to the candidates, which can be ignored when electromagnetic wave is transmitted in the air.

- $T_{f}(i)$ : the $i^{\text {th }}$ forwarding candidate coordination delay which is the time needed for the $i^{\text {th }}$ candidate to acknowledge the sender and suppress other potential forwarders. Note that $T_{f}(i)$ is an increasing function of $i$, since the lower-priority forwarding candidate always needs to wait and confirm that no higher-priority candidates have relayed the packet before it takes its turn to relay the packet.

Thus, the total medium time needed for a packet delivered from the sender to the $i^{\text {th }}$ forwarding candidate is

$$
t_{i}=T_{s}+T_{f}(i)
$$

In the following subsections, we will give examples to illustrate how the candidate prioritization, selection and coordination will affect the expected packet advancement, reliability and medium time cost, which in turn affect the one-hop throughput.

\subsubsection{Impact of Candidate Relay Priority on Through- put}

One factor that will affect the throughput is the candidate relay priority. We use the local forwarding example in Fig.1, and assume $a_{1}$ to $a_{5}$ is normalized to be $1,0.8$, $0.6,0.3$, and 0.1 respectively and $p_{1}$ to $p_{5}$ is $0.1,0.4,0.55$, 0.8 , and 0.9 respectively. We use the IEEE 802.11 DSSS PHY parameter set (in Table 1) to calculate the medium time cost. Assuming data payload size $L_{p l}=512$ bytes and ignoring the propagation delay, $T_{s}, T_{f}(i)$ and $t_{i}$ are:

$$
\begin{aligned}
& T_{s}=T_{D I F S}+T_{h}+L_{p l} / B R=638 \mu s \\
& T_{f}(i)=\left(T_{A C K}+T_{S I F S}\right) i=212 i \mu s \\
& t_{i}=638+212 i \mu s
\end{aligned}
$$

Let's first assume all the available next-hop neighbors are involved in the local forwarding and candidates with larger advancements have higher relay priorities. Assume the sender sends sufficient large number of packets, $N$, then statistically there are $p_{1} N$ number of packets relayed by candidate $s_{1}$ with packet advancement of $a_{1}$ and the corresponding medium time is $t_{1} p_{1} N$. Similarly there are $p_{2}\left(1-p_{1}\right) N$ number of packets relayed by $s_{2}$ with packet advancement of $a_{2}$ and the corresponding medium time cost of $t_{2} p_{2}(1-$ $\left.p_{1}\right) N$. If we define the throughput or transport capacity [7] as the bit-meters successfully transmitted per second. Then totally, there are $L_{p l} \cdot \sum_{i=1}^{5} a_{i}\left(p_{i} N\right) \prod_{w=0}^{i-1}\left(1-p_{w}\right)$ bitmeters are successfully transmitted, and the corresponding medium time cost is $\sum_{i=1}^{5} t_{i}\left(p_{i} N\right) \prod_{w=0}^{i-1}\left(1-p_{w}\right)+t_{5}(N$. $\left.\prod_{w=1}^{4}\left(1-p_{w}\right)\right)$. So from a long term point of view, the one-hop throughput is $2.16 \mathrm{Mbmps}$. However, if we assume the forwarding priority as $s_{2}>s_{3}>s_{4}>s_{5}>s_{1}$, we get the one-hop throughput as $2.34 \mathrm{Mbmps}$, which is larger than the previous case. This result contradicts the common sense that candidates closer to the destination should relay packets first. The reason behind this result is that since the largest-advancement candidate has poor link quality from the sender, in most of the times, it will not receive the packet correctly, but lower-priority candidates always have to wait for a period of time to confirm this situation before they have chances to relay the packet, thus increase the total medium time cost, which in result degrades the throughput.

\subsubsection{Impact of Candidate Selection on Throughput}

Another factor that affects the throughput is the candidate selection. Intuitively, different candidate sets with the same number of forwarding candidates will achieve different throughput. For example, candidate set $\left\langle s_{1}, s_{4}, s_{5}\right\rangle$ achieves throughput of $1.28 \mathrm{Mbmps}$, while candidate set $\left\langle s_{2}, s_{3}, s_{4}\right\rangle$ achieves much higher throughput of $2.35 \mathrm{Mbmps}$. So we should carefully select forwarding candidates that indeed help improve the throughput. Furthermore, different number of forwarding candidates will also result in different throughput. Actually, candidate set $\left\langle s_{2}, s_{3}, s_{4}\right\rangle$ achieves the largest throughput among all the candidate combination and prioritization in this example. When all the available next-hop nodes are involved as forwarding candidates, the throughput does not increase while slightly drops. Therefore, it is unwise to include as many as next-hop neighbors as candidates. Rather, it may be sufficient to just involve a few "good" candidates to achieve the maximum one-hop throughput.

\subsubsection{Impact of Candidate Coordination on Through- put}

The third key factor that will affect the throughput is the candidate coordination delay. Here we use two extreme cases to illustrate the potential impact of this factor on the throughput. First, we assume this delay is negligible, that is, the lower-priority candidates can relay the packet immediately when higher-priority candidates failed to do so. In this case, we should involve all the available next-hop neighbors into opportunistic forwarding, because any extra included candidates would help to improve the relay reliability but without introducing any extra delay. We should also give candidates closer to the destination higher relay priorities, since larger-advancement candidates should always try first in order to maximize the expected packet advancement, even if they were unlikely to receive the packet correctly. If they failed to relay the packet, the lower-priority candidates would instantaneously relay the correctly received 
packet without needing to wait. On the other hand, if the candidate coordination delay is very large comparing to the sender delay, then it is preferable to retransmit the packet in stead of waiting for other forwarding candidates to relay the packet. In this case, one candidate may be optimal. So this factor does affect the throughput, and we will discuss it in more detail in our analysis and simulation.

\subsection{Expected One-hop Throughput (EOT)}

According to the analysis above, for a given forwarding candidate set $\mathcal{F}$, we now propose a new local metric, expected one-hop throughput (EOT) (in Eq. (5)), to characterize the local behavior of GOR in terms of bit-meter advancement per second.

$$
R\left(\mathcal{F}_{j}\right)=L_{p} \cdot \frac{\sum_{i=1}^{r} a_{j_{i}} p_{j_{i}} \cdot \prod_{w=0}^{i-1} \bar{p}_{j_{w}}}{t_{r} \bar{P}_{\mathcal{F}}+\sum_{i=1}^{r} t_{i} p_{j_{i}} \cdot \prod_{w=0}^{i-1} \bar{p}_{j w}}
$$

where $\mathcal{F}_{j}=\left\langle s_{j_{1}}, \ldots, s_{j_{r}}\right\rangle$, which is an ordered set of the nodes in $\mathcal{F}$ with priority $s_{j_{1}}>\ldots>s_{j_{r}} ; p_{j_{0}}:=0 ; \bar{p}_{j_{w}}=1-p_{j_{w}}$; and

$$
\bar{P}_{\mathcal{F}}=\prod_{i=1}^{r}\left(1-p_{i}\right)
$$

which is the probability that none of the forwarding candidates in $\mathcal{F}$ has successfully received the packet in one physical transmission from the sender.

The physical meaning of the EOT defined in Eq. (5) is the expected bit advancement per second for a local GOR procedure. EOT integrates the packet advancement, relay reliability, and MAC medium time cost. The intuitions to maximize EOT are as following: 1) as the whole path achievable throughput is less than per-hop throughput on each link, to maximize the local EOT is likely to increase the path throughput; 2) the path delay is the summation of per-hop delay, which is actually relative to the delay introduced by transmitting the packet and coordinating the candidates. As the per-hop delay factors $\left(T_{s}\right.$ and $\left.T_{f}(i)\right)$ are integrated in the denominators of EOT, to maximize EOT is also implicitly to decrease per-hop delay, which may further decrease the path delay. 3) as EOT also takes into account the packet advancement to the destination, maximizing it potentially decreases hop counts needed to relay the packet to the destination, which may lead to fewer transmissions, alleviated interference to other flows, and decreased delay.

In the following sections, we will examine the behavior of GOR by identifying an upper bound of the EOT and the concavity of the maximum EOT. After that, we will propose a heuristic algorithm to select the forwarding candidates and assign the relay priority to approach an optimal EOT.

\section{UPPER BOUND OF EOT AND ITS CON- CAVITY}

This section studies the performance of GOR in terms of the EOT and we derive an upper bound of EOT.

\subsection{Upper Bound of EOT}

Lemma 1 introduces an upper bound of EOT as follows:

Lemma 1. Given a forwarding candidate set $\mathcal{F}$, the EOT defined in Eq. (5) is upper bounded by $R^{*}$ defined as follows:

$$
R^{*}=L_{p l} \cdot \frac{\sum_{i=1}^{r} a_{i} p_{i} \cdot \prod_{w=0}^{i-1} \bar{p}_{w}}{T_{s}}
$$

Note that $\mathcal{F}$ is indexed according to the advancement s.t. $a_{m} \geq a_{n}, \forall m<n$.
Proof. The minimum value of the denominator of Eq. (5) is obtained when $t_{i}=T_{s}$, i.e. $T_{f}(i)=0$. Denote the numerator of Eq. (5) as

$$
g\left(\mathcal{F}_{j}\right)=\sum_{i=1}^{r} a_{j_{i}} p_{j_{i}} \cdot \prod_{w=0}^{i-1} \bar{p}_{j_{w}}
$$

Now it is sufficient to prove that for any ordered candidate set $\mathcal{F}_{j}$, we have $g\left(\mathcal{F}_{j}\right) \leq g(\mathcal{F})$. This is equivalent to prove that the maximum $g\left(\overline{\mathcal{F}}_{j}\right)$ is obtained by prioritizing the forwarding candidates according to the advancement $a_{j}$, s.t. $a_{m} \geq a_{n} \forall m<n$. We prove this by induction on $r$, i.e., the size of $\mathcal{F}$.

First, for $r=1$, obviously $g\left(\mathcal{F}_{j}\right) \leq g(\mathcal{F})$.

Next, we assume $g\left(\mathcal{F}_{j}\right) \leq g(\mathcal{F})$ holds for $r=\mathrm{M}(\mathrm{M} \geq 1)$, we want to prove it holds for $r=\mathrm{M}+1$.

For $r=\mathrm{M}+1$. $\mathcal{F}$ can be divided into two complementary sub-sets, $\mathcal{A}=\mathcal{F} \backslash\left\{s_{m}\right\}$ with $\mathrm{M}$ nodes and $\mathcal{B}=\left\{s_{m}\right\}$ with 1 node. Then

$$
\begin{aligned}
g\left(\mathcal{F}_{j}\right) & =g\left(\mathcal{A}_{j}\right)+\bar{P}_{\mathcal{A}} \cdot g\left(\left\langle s_{m}\right\rangle\right) \\
& \leq R:=g(\mathcal{A})+\bar{P}_{\mathcal{A}} \cdot g\left(\left\langle s_{m}\right\rangle\right)
\end{aligned}
$$

The first equality holds for the definitions of $g\left(\mathcal{F}_{j}\right)$ and the second inequality holds for the inductive hypothesis. So it suffices to prove $\forall m(1 \leq m \leq M)$, we have $R \leq g(\mathcal{F})$. This can be proved as follows:

$$
g(\mathcal{F})-R=\frac{1}{\bar{p}_{m}} \sum_{k=m+1}^{M+1}\left(a_{m}-a_{k}\right) p_{m} p_{k} \prod_{w=0}^{k-1} \bar{p}_{w} \geq 0
$$

where $\bar{p}_{0}:=1$.

The inequality holds as $a_{m} \geq a_{k} \forall m<k$. So Eq. (7) is an upper bound of Eq. (5) for any given $\mathcal{F}$.

Lemma 1 basically shows that under some idealized MAC scheduling, where the coordination delay among the forwarding candidates is negligible, the maximum EOT can be achieved by giving candidates closer to the destination higher relay priorities.

\subsection{Concavity of the Upper Bound of EOT}

Lemma 1 gives the upper bound of EOT and the corresponding relay priority rule when $\mathcal{F}$ is given. The followed question is how the upper bound changes for different set of $\mathcal{F}$. We answer this question and unveil the concavity of the upper bound of EOT in Theorem 2.

THEOREM 2. Given the available next-hop node set $\mathcal{C}$ with $N(N \geq 1)$ nodes, define $R^{*}(r)$ as the upper bound of the EOT by selecting any $r$ candidates, then $R^{*}(r)$ is an increasing and concave function of $r$.

Proof. ${ }^{2}$ Denote $\mathcal{F}_{r}^{*}$ as the feasible candidate set that achieves $R^{*}(r)$. According to Eq. (7) and (8),

$$
R^{*}(r)=L_{p l} \cdot \frac{g\left(\mathcal{F}_{r}^{*}\right)}{T_{s}}
$$

Then it suffices to prove $g\left(\mathcal{F}_{r}^{*}\right)$ is an increasing and concave function. It's not difficult to see that

$$
g\left(\mathcal{F}_{r+1}^{*}\right) \geq g\left(\left\langle\mathcal{F}_{r}^{*}, s_{m}\right\rangle\right)>g\left(\mathcal{F}_{r}^{*}\right)
$$

where $s_{m} \in \mathcal{C}$ and $s_{m} \notin \mathcal{F}_{r}^{*}$.

\footnotetext{
${ }^{2}$ Due to space limit, we only provide a sketch of the proof.
} 
To prove the concavity of $g\left(\mathcal{F}_{r}^{*}\right)$, we first proved that $\forall$ $\mathcal{F}_{r-1}^{*}, \exists \mathcal{F}_{r}^{*}$, s.t.

$$
\mathcal{F}_{r-1}^{*} \subset \mathcal{F}_{r}^{*} \quad \forall 1 \leq r \leq N
$$

Then according to the containing ${ }^{3}$ property, we assume $\mathcal{F}_{r+1}^{*} \backslash$ $\mathcal{F}_{r}^{*}=\left\{s_{k}\right\}$, and $\mathcal{F}_{r}^{*} \backslash \mathcal{F}_{r-1}^{*}=\left\{s_{j}\right\}$. There are two cases for the advancement relationship between node $s_{k}$ and $s_{j}$.

1) $a_{k}>a_{j}$. Then $\mathcal{F}_{r+1}^{*}, \mathcal{F}_{r}^{*}$ and $\mathcal{F}_{r-1}^{*}$ can be represented as

$$
\begin{aligned}
& \mathcal{F}_{r+1}^{*}=\left\langle\mathcal{A}_{1}, s_{k}, \mathcal{A}_{2}, s_{j}, \mathcal{A}_{3}\right\rangle, \quad \mathcal{F}_{r}^{*}=\left\langle\mathcal{A}_{1}, \mathcal{A}_{2}, s_{j}, \mathcal{A}_{3}\right\rangle, \\
& \mathcal{F}_{r-1}^{*}=\left\langle\mathcal{A}_{1}, \mathcal{A}_{2}, \mathcal{A}_{3}\right\rangle
\end{aligned}
$$

where $\mathcal{A}_{i}(1 \leq i \leq 3)$ is ordered node set and can be $\emptyset$. With

$$
\mathrm{B}:=g\left(\mathcal{F}_{r}^{*}\right)-g\left(\left\langle\mathcal{A}_{1}, s_{k}, \mathcal{A}_{2}, \mathcal{A}_{3}\right\rangle\right) \geq 0
$$

we have

$$
\begin{aligned}
& {\left[g\left(\mathcal{F}_{r}^{*}\right)-g\left(\mathcal{F}_{r-1}^{*}\right)\right]-\left[g\left(\mathcal{F}_{r+1}^{*}\right)-g\left(\mathcal{F}_{r}^{*}\right)\right]} \\
& =\mathrm{B}+\left(1-p_{\mathcal{A}_{1}}\right)\left(1-p_{\mathcal{A}_{2}}\right) p_{k} p_{j}\left(a_{j}-g\left(\mathcal{A}_{3}\right)\right)>0
\end{aligned}
$$

where $p_{\mathcal{A}_{i}}$ is the probability of at least one node in $\mathcal{A}_{i}$ receives the packet correctly.

Inequality (13) holds because $\mathrm{B} \geq 0$ (inequality (12)) and $a_{j}-g\left(\mathcal{A}_{3}\right)>0$.

2) $a_{k}<a_{j}$. Similarly,

$$
\begin{aligned}
\mathcal{F}_{r+1}^{*} & =\left\langle\mathcal{A}_{1}, s_{j}, \mathcal{A}_{2}, s_{k}, \mathcal{A}_{3}\right\rangle, \quad \mathcal{F}_{r}^{*}=\left\langle\mathcal{A}_{1}, s_{j}, \mathcal{A}_{2}, \mathcal{A}_{3}\right\rangle, \\
\mathcal{F}_{r-1}^{*} & =\left\langle\mathcal{A}_{1}, \mathcal{A}_{2}, \mathcal{A}_{3}\right\rangle
\end{aligned}
$$

With

$$
\mathrm{B}:=g\left(\mathcal{F}_{r}^{*}\right)-g\left(\left\langle\mathcal{A}_{1}, \mathcal{A}_{2}, s_{k}, \mathcal{A}_{3}\right\rangle\right) \geq 0
$$

we have

$$
\begin{aligned}
& {\left[g\left(\mathcal{F}_{r}^{*}\right)-g\left(\mathcal{F}_{r-1}^{*}\right)\right]-\left[g\left(\mathcal{F}_{r+1}^{*}\right)-g\left(\mathcal{F}_{r}^{*}\right)\right]} \\
& =\mathrm{B}+\left(1-p_{\mathcal{A}_{1}}\right)\left(1-p_{\mathcal{A}_{2}}\right) p_{k} p_{j}\left(a_{k}-g\left(\mathcal{A}_{3}\right)\right)>0
\end{aligned}
$$

From the analysis above, we know $R^{*}(r)$ is an increasing and concave function of $r$.

Theorem 2 indicates that even if the coordination delay among the forwarding candidates were negligible, the gained throughput by increasing the number of the forwarding candidates would become marginal. So it may only need to involve a small number of forwarding candidates to achieve the best EOT.

\section{HEURISTIC CANDIDATE SELECTION ALGORITHM}

A straightforward way to get the optimal $\mathcal{F}$ and the corresponding $\mathcal{F}_{j}$ to maximize the EOT is to try all the ordered subset of $\mathcal{C}$, which runs in $\Omega(N$ !) time, where $N$ is the number of available next-hop nodes. It is, however, not feasible when $N$ is large. In this section, we propose a heuristic candidate selection and prioritization algorithm to get a solution approaching the optimal EOT.

By observing Eq. (5), we can find that the candidate achieving the maximum EOT by selecting 1 node from $\mathcal{C}$ is contained in at least one feasible candidate set achieving the maximum EOT by selecting $r(1 \leq r \leq|\mathcal{C}|)$ nodes from

\footnotetext{
${ }^{3}$ In this paper, an ordered node set $\mathcal{A}$ containing another ordered node set $\mathcal{B}$ means $\mathcal{A}$ is obtained by inserting a new node into $\mathcal{B}$ but keeping the priority relationship of nodes in $\mathcal{B}$ unchanged. It's not necessary for $\mathcal{B}$ being a subsequence of $\mathcal{A}$.
}

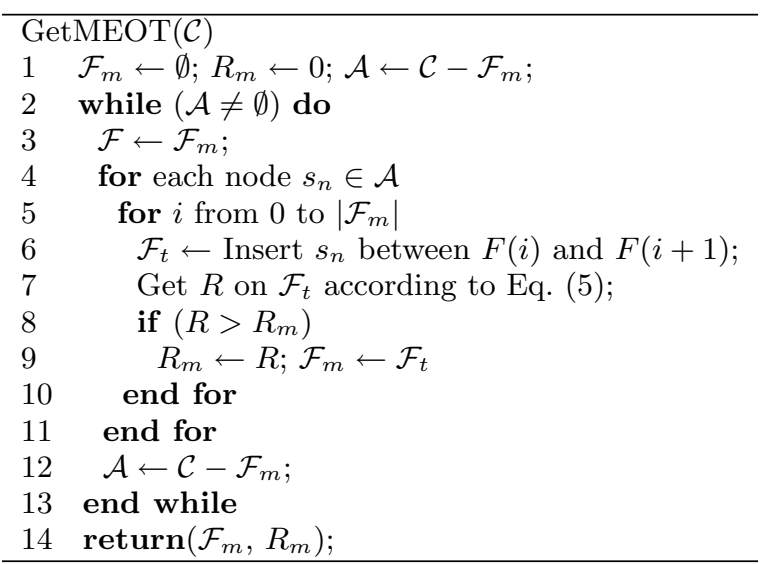

Table 2: Pseudocode of finding an ordered candidate set $\mathcal{F}_{m}$, and the corresponding $R_{m}$ for a given available next-hop set $\mathcal{C}$

$\mathcal{C}$. Because if it were not the case, we could always substitute the lowest-priority node in the optimal set (with $r$ nodes) to get another new candidate set which achieves an EOT no smaller than that of the optimal set, which is a contradiction. Then, we propose the algorithm GetMEOT in Table 2 which finds an $\mathcal{F}$ based on this observation. This algorithm greedily adds a new node into the current optimal/suboptimal $\mathcal{F}$ containing $r$ nodes without changing the priorities among the $\mathrm{r}$ nodes to get an optimal/suboptimal $\mathcal{F}$ with $r+1$ nodes. Finally, the candidate set with the maximum EOT is returned. This algorithm runs in $O\left(|\mathcal{C}|^{3}\right)$. An interesting result is that this algorithm almost surely finds the actual global optimal $\mathcal{F}$ in our simulation.

\section{PERFORMANCE EVALUATION}

We validate the concavity of the upper bound of EOT and evaluate the one-hop performance as well as the path performance of GOR that applies the GetMEOT algorithm by simulation. We compare the GOR with the geographic routing which selects one neighbor with maximum $a_{j} p_{j}[10$, $9]$, and the pure opportunistic routing which involves all the available next-hop nodes with nodes closer to the destination having higher relay priorities.

\subsection{Simulation Setup}

We assume $T_{s}=T_{\text {backoff }}+T_{D I F S}+T_{h}+L_{p l} / B R$ and $T_{f}(i)=\left(T_{A C K}+T_{S I F S}\right) i$, which are calculated according to Table 1 , by assuming $L_{p l}=512$ bytes. The simulated network has stationary nodes uniformly distributed in a $1200 \times 1200 \mathrm{~m}^{2}$ square region with nodes having identical transmission power of $15 \mathrm{dbm}$. The source and the destination nodes are fixed at two corners across the diagonal of the square area. We also assume an ideal collision-free MAC such that packet loss is only due to the randomness of link quality, and at any time there is only one transmission scheduled. The results are averaged from 200 runs, and in each run, there are 2000 packets delivered to the destination. To investigate the impact of node density on the performance of these routing schemes, we vary the number of nodes as $35,50,80,100$, which corresponds to different node densities as 11, 16, 22, 34 neighbors per node.

We use the Nakagami distribution [12] to describe the 


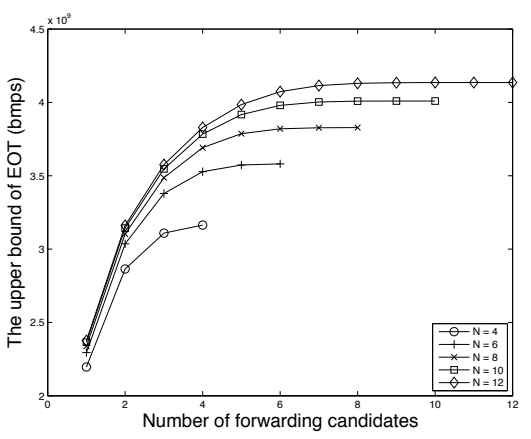

Figure 2: The upper bound of EOT under different number of available next-hop neighbors.

power $\mathrm{x}$ of a received signal:

$$
f(x ; m, \Omega)=\frac{m^{m} x^{m-1}}{\Gamma(m) \Omega^{m}} \exp \left(-\frac{m x}{\Omega}\right)
$$

where $\Gamma$ is the Gamma function, $m$ denotes the Nakagami fading parameter and $\Omega$ is the average received power. We set $m=1$ in our simulation. Assuming two-ray signal propagation, $\Omega$ can be expressed in Eq. (17) as a function of $d$, the distance between the sender and receiver.

$$
\Omega(d)=\frac{P_{t} G_{t} G_{r} h_{t}^{2} h_{r}^{2}}{d^{n}}
$$

where $P_{t}$ is the transmission power, $G_{t}$ and $G_{r}$ the antenna gains, $h_{t}$ and $h_{r}$ the antenna heights, and $n$ the path-loss exponent. We set $G_{t}=G_{r}=1, h_{t}=h_{r}=1.5 \mathrm{~m}$, and $n=4$ in our simulation.

We assume a packet is received successfully if the received signal power is greater than the receiving power threshold. According to $802.11 \mathrm{~b}[1]$, the threshold for $11 \mathrm{Mbps}$ data rate is $-82 \mathrm{dbm}$. Then by using Eq. (16) and (17), we can derive the PRR at a certain distance $d$.

\subsection{Evaluation Metrics}

We define the following evaluation metrics:

- One-hop throughput: number of bit-meters successfully delivered per second medium time in one-hop with unit of bmps.

- Path throughput: number of bit-meters successfully delivered per second from the source to the destination in the whole duration of simulation with unit of bmps.

\subsection{Simulation Results and Analysis}

\subsubsection{Concavity of the upper bound of EOT}

In this subsection, we study the concavity of the upper bound of EOT. The nodes are uniformly distributed and the size of the available next-hop nodes is various from 4 to 12. From Fig. 2 we can see that the upper bound of EOT increases when the number of the forwarding candidates increases, and when nodes are denser ( $\mathrm{N}$ is larger), the EOT is larger. A very interesting result is that under different node densities, the shapes of all the curves are nearly the same, which manifests that no matter what the node density is, the gained EOT of involving more forwarding candidates becomes marginal. The slopes of all the curves approach to

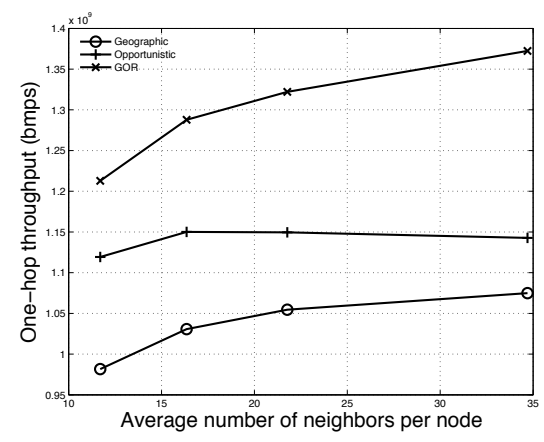

Figure 3: One-hop throughput under various node densities

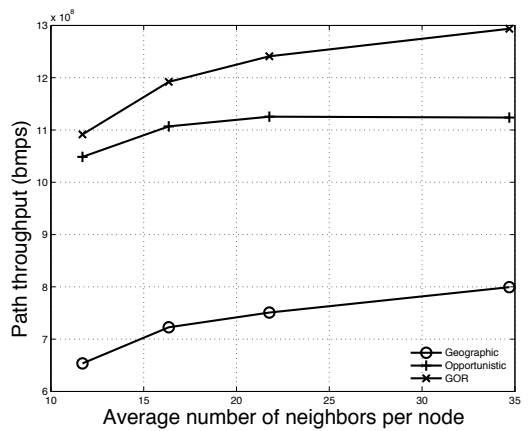

Figure 4: Path throughput under various node densities

0 when the number of forwarding candidates is larger than 4 .

\subsubsection{One-hop and path performance}

The one-hop throughput of the three protocols under different node densities are shown in Fig. 3. We see that, our scheme (GOR) achieves the best one-hop throughput in the three schemes. The pure opportunistic routing that involves all the available next-hop nodes into the local forwarding is not optimal. The geographic routing that only includes one forwarding candidate performs worst, because it has the lowest per hop transmission reliability, then need more physical retransmissions to make a successful data delivery at the network layer.

Fig. 4 shows the path throughput of the three routing schemes, which represents the same trend as the one-hop performance in Fig. 3. This indicates that the per-hop optimization of EOT also implies optimal path throughput.

\section{RELATED WORK}

\subsection{Geographic Routing}

A key advantage of geographic routing is that the nodes are not required to maintain extensive routing tables, and can make simple routing decisions based on the local geographic position of its neighboring nodes. More recent works on geographic routing are focused on lossy channel situations [10, 17, 9, 14]. Seada, et al. [10] concluded that packet advancement timing packet reception ratio is an optimal metric for making localized geographic routing decisions 
in lossy wireless networks with ARQ (Automatic Repeat reQuest) mechanisms. Zorzi and Armaroli also independently proposed the same link metric [17]. Lee et al. [9] presented a more general framework to normalize various types of link cost such as transmission times, delay and power consumption. Unfortunately, that framework only applies to geographic routing which involves single forwarding candidate and can not be directly used for GOR.

\subsection{Opportunistic routing}

Some variants of opportunistic routing, such as ExOR [3] and opportunistic any-path forwarding [16], rely on the path cost information or global knowledge of the network to select candidates and prioritize them. In the least-cost opportunistic routing (LCOR) [5], it needs to enumerate all the neighboring node combinations to get the least cost OR paths. While some other variants $[18,11,6]$ use the location information of nodes to define the candidate set and relay priority. In GeRaF [18], the next-hop neighbors of the current forwarding node are divided into sets of priority regions with nodes closer to the destination having higher relay priorities. The energy performance is analyzed in [18]. One similar work to [18] is [11] where the network layer specifies a set of nodes by defining a forwarding region in space that consists of the candidate nodes and the data link layer selects the first node available from that set to be the next hop node. [6] discussed three suppression strategies of contention-based forwarding to avoid packet duplication in mobile ad hoc networks. Our work belongs to the second kind of variants, but provides more insightful understanding of the trade-off among the packet advancement, coordination time cost and reliability associated with the node collaboration.

\section{CONCLUSION}

In this paper, we study the geographic opportunistic routing (GOR) scheme, and analyze the trade-off among the packet advancement, reliability and MAC coordination time cost in GOR. We introduce a new local routing metric, the expected one-hop throughput (EOT), to balance these factors. We then derive an upper bound of the EOT, and unveil its concavity, which indicates that although involving more forwarding candidates brings more chances for the packet to get closer to the destination and be delivered, the gained benefit becomes marginal when we are doing so. The EOT upper bound analysis also manifests that if the coordination delay among the forwarding candidates were negligible, the maximum EOT could be achieved by giving candidates closer to the destination higher relay priorities. Based on EOT, we further propose a heuristic algorithm to select the forwarding candidates and prioritize them. The simulation results validate our analysis and show that GOR achieves higher one-hop throughput as well as path throughput than the corresponding pure opportunistic routing and geographic routing schemes.

\section{REFERENCES}

[1] IEEE Std 802.11b-1999.

[2] B. Awerbuch, D. Holmer, and H. Rubens. The medium time metric: High throughput route selection in multi-rate ad hoc wireless networks. MONET, 11(2):253-266, 2006.
[3] S. Biswas and R. Morris. Exor: Opportunistic multi-hop routing for wireless networks. In SIGCOMM'05, Philadelphia, Pennsylvania, Aug. 2005.

[4] D. Couto, D. Aguayo, J. Bicket, and R. Morris. A high-throughput path metic for multi-hop wireless routing. In ACM MobiCom'03, San Diego, California, Sept. 2003.

[5] H. Dubois-Ferriere, M. Grossglauser, and M. Vetterli. Least-cost opportunistic routing. Technical Report LCAV-REPORT-2007-001, School of Computer and Communication Sciences, EPFL, 2007.

[6] H. Fussler, J. Widmer, M. Kasemann, M. Mauve, and H. Hartenstein. Contention-based forwarding for mobile ad-hoc networks. Elsevier's Ad Hoc Networks, 1(4):351-369, Nov. 2003.

[7] P. Gupta and P. R. Kumar. The capacity of wireless networks. Trans. Inform. Theory, 46(2):388-404, Mar. 2000.

[8] A. Keshavarzin, E. Uysal-Biyikoglu, F. Herrmann, and A. Manjeshwar. Energy-efficient link assessment in wireless sensor networks. In IEEE Infocom, March 2004.

[9] S. Lee, B. Bhattacharjee, and S. Banerjee. Efficient geographic routing in multihop wireless networks. In MobiHoc, 2005.

[10] K. Seada, M. Zuniga, A. Helmy, and B. Krishnamachari. Energy efficient forwarding strategies for geographic routing in wireless sensor networks. In ACM Sensys'04, Baltimore, MD, Nov. 2004.

[11] R. C. Shah, A. Bonivento, D. Petrovic, E. Lin, J. van Greunen, and J. Rabaey. Joint optimization of a protocol stack for sensor networks. In IEEE Milcom, Nov. 2004.

[12] M. K. Simon and M.-S. Alouini. Digital communication over fading channels. Wiley-Interscience, 2nd edition, 2005.

[13] K. Zeng, W. Lou, J. Yang, and D. R. Brown. On geographic collaborative forwarding in wireless ad hoc and sensor networks. In WASA'07, Chicago, IL, August 2007.

[14] K. Zeng, K. Ren, W. Lou, and P. J. Moran. Energy aware efficient geographic routing in lossy wireless sensor networks with environmental energy supply. Wireless Networks (WINET), 2007.

[15] J. Zhao and R. Govindan. Understanding packet delivery performance in dense wireless sensor networks. In ACM Sensys'03, LA,CA, Nov. 2003.

[16] Z. Zhong, J. Wang, and S. Nelakuditi. Opportunistic any-path forwarding in multi-hop wireless mesh networks. Technical Report TR-2006-015, USC-CSE, 2006.

[17] M. Zorzi and A. Armaroli. Advancement optimization in multihop wireless networks. Proc. of $V T C$, Oct. 2003.

[18] M. Zorzi and R. R. Rao. Geographic random forwarding (geraf) for ad hoc and sensor networks: energy and latency performance. IEEE Transactions on Mobile Computing, 2(4), 2003. 